\title{
Avaliação dos conhecimentos cognitivos associados ao debriefing na simulação
}

\section{realística}

\author{
Assessment of cognitive knowledge associated with debriefing in realistic simulation \\ Evaluación del conocimiento cognitivo asociado al debriefing en simulación realista
}

Recebido: 04/02/2022 | Revisado: 13/02/2022 | Aceito: 19/02/2022 | Publicado: 28/02/2022

\author{
Luciana de Melo Mota \\ ORCID: https://orcid.org/0000-0001-8745-9292 \\ Centro Universitário Tiradentes, Brasil \\ E-mail: lummota@hotmail.com \\ Ayra Lisiane Ferreira dos Santos \\ ORCID: https://orcid.org/0000-0002-0896-845X \\ Centro Universitário Tiradentes, Brasil \\ E-mail: ayra.lisiane@gmail.com \\ Rozangela Maria de Almeida Fernandes Wyszomirska \\ ORCID: https://orcid.org/0000-0003-0066-8927 \\ Universidade Estadual de Ciências da Saúde de Alagoas, Brasil \\ E-mail: rozangela.almeida@uncisal.edu.br
}

\begin{abstract}
Resumo
O desenvolvimento de competências pelos estudantes está intimamente ligado à utilização de novas modalidades de ensino, fator que desperta mais interesse por parte dos estudantes. Nesse sentido, a Simulação Realística (RS) surge como uma forma de metodologia ativa que estimula o processo de ensino-aprendizagem. Nessa perspectiva, este estudo busca responder à seguinte questão de pesquisa: quais são as formas de avaliar o conhecimento cognitivo de estudantes de medicina durante um debriefing em simulação realista? Com o objetivo de avaliar o conhecimento cognitivo de estudantes de medicina associado a um debriefing em simulação realística, buscando evidenciar o tema abordado. Trata-se de um estudo descritivo com abordagem quantitativa realizado no Centro de Simulação (CSIM) do Centro Universitário Tiradentes-UNIT / AL, localizado no Hospital Veredas, equipado com manequins de alta fidelidade (adulto, gestante e infantil) para simulação realística. Foi utilizado como instrumento de coleta de dados a Escala de Avaliação de Debriefing associada à Simulação (EADaS) e participaram deste estudo 99 estudantes, 61 $(61,6 \%)$ do sexo feminino e 38 (38,4\%) do masculino, nos cenários de Urgência e Emergência, Obstetrícia e Pediatria. No que diz respeito aos aspectos cognitivos, destaca-se a estruturação do pensamento, relacionado à aprendizagem, foco nos aspectos mais importantes da experiência simulada, aprofundamento de conhecimentos específicos relacionados à simulação e a capacidade de identificar o que deve ser melhorado em futuras situações de simulação. $\mathrm{O}$ debriefing mostrou-se uma metodologia de avaliação capaz de estimular o desenvolvimento de competências e habilidades necessárias ao exercício profissional da medicina.
\end{abstract}

Palavras-chave: Simulação realística; Debriefing; Estudantes de medicina; Cognição.

\begin{abstract}
The development of competences by students is closely linked to the use of new teaching modalities, a factor that arouses more interest on the part of students. In this sense, Realistic Simulation (SR) emerges as a form of active methodology that stimulates the teaching-learning process. In this perspective, this study seeks to answer the following research question: what are the ways to assess the cognitive knowledge of medical students during a debriefing in a realistic simulation? With the objective of evaluating the cognitive knowledge of medical students associated with a debriefing in realistic simulation, seeking to highlight the topic addressed. This is a descriptive study with a quantitative approach carried out at the Simulation Center (CSIM) of Centro Universitário Tiradentes-UNIT / AL, located at Hospital Veredas, equipped with high-fidelity mannequins (adult, pregnant and child) for realistic simulation. The Debriefing Assessment Scale associated with Simulation (EADaS) was used as a data collection instrument and 99 students participated in this study, 61 (61.6\%) female and $38(38.4 \%)$ male, in the scenarios and Emergency, Obstetrics and Pediatrics. With regard to cognitive aspects, we highlight the structuring of thinking, related to learning, focus on the most important aspects of the simulated experience, deepening of specific knowledge related to simulation and the ability to identify what should be improved in future situations of simulation. The debriefing proved to be an assessment methodology capable of stimulating the development of skills and abilities necessary for the professional practice of medicine.
\end{abstract}

Keywords: Realistic simulation; Debriefing; Medical students; Cognition. 


\section{Resumen}

El desarrollo de competencias por parte de los estudiantes está íntimamente ligado al uso de nuevas modalidades de enseñanza, factor que despierta mayor interés por parte de los estudiantes. En este sentido, la Simulación Realista (SR) surge como una forma de metodología activa que dinamiza el proceso de enseñanza-aprendizaje. En esta perspectiva, este estudio busca responder a la siguiente pregunta de investigación: ¿cuáles son las formas de evaluar el conocimiento cognitivo de los estudiantes de medicina durante un debriefing en una simulación realista? Con el objetivo de evaluar el conocimiento cognitivo de estudiantes de medicina asociado a un debriefing en simulación realista, buscando resaltar el tema abordado. Se trata de un estudio descriptivo con abordaje cuantitativo realizado en el Centro de Simulación (CSIM) del Centro Universitário Tiradentes-UNIT/AL, ubicado en el Hospital Veredas, equipado con maniquíes de alta fidelidad (adulto, gestante y niño) para simulación realista. La Escala de Evaluación del Debriefing asociada a la Simulación (EADaS) fue utilizada como instrumento de recolección de datos y participaron de este estudio 99 estudiantes, 61 (61,6\%) del sexo femenino y $38(38,4 \%)$ del sexo masculino, en los escenarios y Emergencia, Obstetricia y Pediatría. En cuanto a los aspectos cognitivos, destacamos la estructuración del pensamiento, relacionado con el aprendizaje, el enfoque en los aspectos más importantes de la experiencia simulada, la profundización de conocimientos específicos relacionados con la simulación y la capacidad de identificar lo que se debe mejorar en futuras situaciones de simulación. El debriefing demostró ser una metodología de evaluación capaz de estimular el desarrollo de habilidades y destrezas necesarias para el ejercicio profesional de la medicina.

Palabras clave: Simulación realista; Interrogación; Estudiantes de medicina; Cognición.

\section{Introdução}

No ensino em saúde, uma série de inovações, inclusões de novas metodologias de ensino-aprendizagem apontam para a adoção de metodologias ativas, exigindo que o docente repense, reavalie e reconstrua sua prática pedagógica, buscando novas estratégias de ensino, que devem ser capazes de melhorar os resultados de aprendizagem e que ofereçam subsídios para a formação de indivíduos críticos-reflexivos, com autonomia de pensar suas ações e escolhas, capazes de transformar a si e a seu contexto (Waterkemper et al., 2011).

Ressalta-se que o ensino através da simples transmissão de informação, sem algo prático, não pode mais ser caracterizado como uma forma de ensino-aprendizagem eficiente. Desse modo, os discentes necessitam de novas estratégias que sejam capazes de melhorar o desempenho cognitivo, o estímulo à autoconfiança e a promoção de satisfação. Assim, o desenvolvimento de habilidades por estudantes está intimamente ligado à utilização de novas modalidades de ensino, fator que gera mais interesse por parte dos discentes (Lovato et al., 2018).

A Simulação Realística (SR) vem emergindo como forma de metodologia ativa que estimula o processo de ensinoaprendizagem, tendo em vista que contempla esse processo de forma significativa, reproduzindo diferentes realidades no ensino em saúde (Mazzo et al., 2019). O uso da simulação atende às necessidades do ensino da prática teórica e da formação atual, e é valorizada pelos estudantes, melhorando sua consciência e percepção psicomotora da realidade da prática clínica que os espera e precisa se preocupar (Marcomini et al.,2017). Nesse sentido, a SR pode ser entendida como uma metodologia ativa que replica cenários de cuidados ao paciente em um ambiente próximo à realidade, com o objetivo de analisar e refletir as ações realizadas de forma segura. Além de ser uma metodologia poderosa de aprendizado, pode ser aplicada em todos os níveis da educação médica, enfatizando a multidisciplinaridade em diversas situações clínicas (Brandão et al., 2014; Ypinazar et al., 2006).

"Na simulação, os elementos partícipes são: o facilitador; os participantes; práticas educacionais (caso clínico e a simulação clínica); características do desenho de simulação (com foco em resolução de problemas) e expectativa dos resultados das simulações (debriefing)" (Costa et al., 2020, pp.2). Avalia-se que o debriefing constitui a última etapa de um ambiente de SR e é a mais importante, visto que é nesse processo que o estudante tem a oportunidade de refletir sobre suas ações e desenvolver a habilidade de pensar criticamente em meio a situações que exigem um raciocínio rápido. Além disso, ter um conhecimento consistente sobre teoria e prática permite um melhor momento de reflexão sobre os exercícios, há uma melhora da conexão entre teoria e situações práticas na vida real, maior domínio de informações, sendo útil para sanar dúvidas 
e promover uma reflexão sobre tudo que é abordado durante a SR e, desse modo, proporciona um melhor desenvolvimento cognitivo, afetivo e psicossocial (Ferreira et al., 2018; Rodrigues et al., 2019).

Neste estudo, o conceito de conhecimento cognitivo utilizado parte da perspectiva teórica da Pirâmide de Miller. O "sabe", base da pirâmide, se refere a avaliação de como os estudantes integram os conhecimentos prévios com a nova informação: o saber. O conhecimento, na perspectiva teórica, é de fundamental relevância para que o estudante possa atingir outros níveis da pirâmide: o "sabe como", o "mostra como", e o "faz". Assim, a avaliação de habilidades cognitivas pode ser feita através de medidas educacionais, como por exemplo o uso da SR (Miller, 1990). Estimular o envolvimento dos estudantes da saúde com a execução da SR proporciona um progresso intelectual e cognitivo, melhora da sistematização dos procedimentos e diminuição de erros futuros. Conforme se observa, a utilização da SR permite um maior domínio de habilidades pelos estudantes, derivado da reprodução contínua das atividades de uma forma segura (Vaccari et al., 2020). A prática da SR ajuda tanto os discentes que estão praticando, quanto os docentes que estão aplicando as atividades em meio aos cenários de simulação (Valadares \& Magro, 2014). Nessa direção, avalia-se que, para os estudantes, há uma melhora da conexão entre teoria e situações práticas na realidade, maior domínio de informações, útil para sanar dúvidas e promover uma reflexão sobre tudo que é abordado durante a SR (Rodrigues et al., 2019).

Nessa perspectiva, o presente estudo busca responder a seguinte questão de pesquisa: quais são as formas de avaliar o conhecimento cognitivo de estudantes de medicina durante um debriefing em simulação realística? buscando evidenciar o tema abordado, a fim de melhorar a compreensão dos professores ou facilitadores acerca do desenvolvimento dessas habilidades intelectuais dos discentes, além de identificar as formas de avaliação cognitiva.

\section{Metodologia}

Trata-se de um estudo descritivo com abordagem quantitativa. A pesquisa descritiva descreve situações ou indivíduos de forma detalhada, de modo que permite abranger as características dessas situações e indivíduos. Tem por finalidade observar e estabelecer uma relação entre as variáveis determinadas e o objeto de estudo (Pedroso et al., 2018). Já os estudos quantitativos são caracterizados pela coleta e análise de dados quantitativos sobre variáveis, sendo possível identificar a realidade de modo mais exato, determinar fatores de associação entre as variáveis e explicar os motivos dos acontecimentos (Esperón, 2017). O estudo foi realizado no Centro de Simulação (CSIM) do Centro Universitário Tiradentes-UNIT/AL, localizado no Hospital Veredas, equipado com manequins de alta fidelidade (adulto, gestante e criança) para simulação realística, dispondo ainda de 1 enfermaria, 2 auditórios e 2 salas de debriefing, para que os estudantes pudessem acompanhar o debriefing.

A amostra do estudo foi obtida por conveniência e constituída de estudantes do $10^{\circ}, 11^{\circ}$ e $12^{\circ}$ períodos do curso de medicina do Centro Universitário Tiradentes, formado por 132 estudantes, ambos os gêneros e todas as faixas etárias. O cálculo teve $95 \%$ de grau de confiança, sendo 99 estudantes considerados uma amostra representativa para o universo. Os critérios de inclusão foram todos os estudantes de medicina do $10^{\circ}, 11^{\circ}$ e $12^{\circ}$ períodos que estivessem regularmente matriculados no curso de Medicina do Centro Universitário Tiradentes-UNIT/AL e que atendessem os seguintes critérios de elegibilidade: tivessem frequentado pelo menos quatro aulas de simulação realística, e participado do debriefing, que corresponde a $50 \%$ das aulas de acordo com o cronograma da instituição. Já os critérios de exclusão foram os estudantes afastados por licença médica no período da coleta de dados e os que se recusaram em assinar o Termo de Compromisso Livre e Esclarecido (TCLE).

Ao término da aula de simulação realística, após o debriefing, os estudantes foram convidados a responder a Escala de Avaliação do Debriefing associado à Simulação (EADaS). Utilizado como instrumento para a coleta de dados, a EADaS, que foi validada por uma banca de juízes e possui 34 itens, sendo construída e validada por Coutinho et al. (2014). Sua 
confiabilidade foi mensurada, demonstrando o coeficiente de alfa de Cronbach de 0,899 (Almeida et al., 2016). Os itens foram divididos por três dimensões: a dimensão valor psicossocial, a dimensão valor cognitivo e a dimensão valor afetivo. Conforme proposto por Coutinho et al. (2014): "No quadro seguinte são realizadas várias afirmações relativas à discussão que fizemos após a prática simulada (debriefing). Para cada uma, assinale a sua opinião. Não há respostas certas ou erradas. Responda por favor de acordo com a sua verdadeira opinião".

Inicialmente foi solicitado à coordenação do curso de medicina do Centro Universitário Tiradentes-UNIT/AL e ao Centro de Estudos do Hospital Veredas a autorização institucional para a realização do estudo. Após a autorização destes, o projeto foi submetido e aprovado pelo Comitê de Ética em Pesquisa (CEP) através da Plataforma Brasil, com o parecer de $\mathrm{n}^{\circ}$ 4.014.682. Após aprovação do projeto de pesquisa pelo CEP, foi solicitado à coordenação do curso de medicina, uma relação dos estudantes que estavam matriculados no internato, com o intuito de selecionar a amostra dos participantes. O cálculo amostral utilizou uma margem de confiança de 95\%, para uma população mais homogênea, e uma margem de erro de 5\% de acordo com os estudantes que aceitaram participar do estudo. A amostra foi composta por 99 estudantes de graduação em medicina dos três últimos períodos do internato. Nessa direção, as questões foram submetidas ao teste de hipóteses foram submetidas ao teste de hipóteses de qui-quadrado $\left(\chi^{2}\right)$ para verificação de diferença significativa em nível de $5 \%$.

\section{Resultados}

Participaram deste estudo 99 estudantes, sendo 61 (61,6\%) do sexo feminino e $38(38,4 \%)$ do sexo masculino, que realizaram atividades práticas nos cenários das disciplinas de Urgência e Emergência, Obstetrícia e Pediatria, no período de 4 meses: novembro e dezembro de 2020 e janeiro e fevereiro de 2021.

A EADaS, no que diz respeito aos aspectos da dimensão cognitiva, possuem 9 perguntas associadas com as recomendações das Diretrizes Curriculares Nacionais dos Cursos de graduação em Medicina (DCNs).

Para a dimensão Cognitiva, os itens P1 "Estruturar meu pensamento", P4 "Me focar nos aspectos mais importantes da atuação", P6 "Refletir sobre as minhas competências", P7 "Identificar prioridades na atuação", P10 "Aprofundar conhecimentos específicos relacionados com a atuação" e P13 "Desenvolver competências para tomada de decisões acertadas" apresentaram uma média de concordância de 98,4\%, e, vale destacar, que os itens P3 “Aprender mais" e P12 "Identificar aspectos que devo melhorar em atuações futuras" obtiveram um índice de concordância de 99,4\% (Tabela 1).

Tabela 1. Distribuição dos itens da Escala de Avaliação do Debriefing associado a Simulação de acordo com a dimensão cognitiva.

\begin{tabular}{|c|c|c|c|c|c|c|c|c|c|c|}
\hline \multirow[t]{2}{*}{ Dimensão Cognitiva } & \multirow{2}{*}{$\begin{array}{c}\begin{array}{c}\text { Discordo } \\
\text { completamente }\end{array} \\
\mathbf{N} \\
\end{array}$} & \multicolumn{3}{|c|}{ Discordo } & \multicolumn{2}{|c|}{ Indiferente } & \multicolumn{2}{|c|}{ Concordo } & \multicolumn{2}{|c|}{$\begin{array}{c}\text { Concordo } \\
\text { completamente }\end{array}$} \\
\hline & & $\%$ & $\mathbf{N}$ & $\%$ & $\mathbf{N}$ & $\%$ & $\mathbf{N}$ & $\%$ & $\mathbf{N}$ & $\%$ \\
\hline 1-Estruturar meu pensamento & 1 & 1,0 & 0 & & 1 & 1,0 & 25 & 25,2 & 72 & 72,7 \\
\hline 3-Aprender mais & 0 & & 0 & & 1 & 1,0 & 13 & 13,1 & 85 & 85,8 \\
\hline
\end{tabular}

\begin{tabular}{|c|c|c|c|c|c|c|c|c|c|c|}
\hline $\begin{array}{c}\text { 4-Me focar nos aspectos mais } \\
\text { importantes da atuação }\end{array}$ & 0 & & 0 & & 1 & 1,0 & 27 & 27,2 & 71 & 71,7 \\
\hline $\begin{array}{c}\text { 6-Refletir sobre as minhas } \\
\text { competências }\end{array}$ & 0 & & 1 & 1,0 & 0 & & 28 & 28,2 & 70 & 70,7 \\
\hline $\begin{array}{c}\text { 7-Identificar prioridades na } \\
\text { atuação }\end{array}$ & 0 & & 0 & & 0 & 2,0 & 25 & 25,2 & 72 & 72,7 \\
\hline
\end{tabular}




\begin{tabular}{|c|c|c|c|c|c|c|c|c|c|c|}
\hline $\begin{array}{c}\text { 8-Melhor identificar os recursos a } \\
\text { utilizar na atuação }\end{array}$ & 0 & & 0 & & 6 & 6,0 & 32 & 32,3 & 61 & 61,6 \\
\hline $\begin{array}{c}\text { 10-Aprofundar conhecimentos } \\
\text { específicos relacionados com a } \\
\text { atuação }\end{array}$ & 0 & & 0 & & 2 & 2,0 & 27 & 27,2 & 70 & 70,7 \\
\hline $\begin{array}{c}\text { 12-Identificar aspectos que devo } \\
\text { melhorar em atuações futuras }\end{array}$ & 0 & & 0 & & 0 & & 19 & 19,1 & 80 & 80,8 \\
\hline $\begin{array}{c}\text { 13-Desenvolver competências para } \\
\text { tomada de decisões acertadas }\end{array}$ & 0 & & 0 & 1,0 & 0 & & 24 & 24,2 & 74 & 74,7 \\
\hline
\end{tabular}

Fonte: Dados da pesquisa (2021).

No que se refere aos aspectos cognitivos, os valores de $\chi 2 \geq 2,0$ demonstraram que a hipótese nula deve ser rejeitada para as categorias das questões P3, P4 P10 e P12, com diferença significativa entre elas (P<0,05), ou seja a estruturação do pensamento, relacionada com aprendizagem, foco nos aspectos mais importantes da experiência simulada, aprofundamento de conhecimentos específicos relacionados a simulação e capacidade de identificar o que deve ser melhorado em situações de simulações futuras. A hipótese nula deve ser retida para P6, P7, P8 e P13; portanto, sem diferença significativa (p>0.05), para as questões relacionadas a reflexão das competências, identificação de prioridade na experiência simulada, identificação de recursos e o desenvolvimento de competências para tomada de decisões.

Tabela 2. Comparação entre os valores de respostas dos aspectos cognitivos da Escala de Avaliação do Debriefing associado à Simulação.

\begin{tabular}{|c|c|c|}
\hline Dimensão cognitiva & Teste qui-quadrado & p- valor* \\
\hline P1 X P3 & 9,900 & $\mathrm{P}=, 002$ \\
\hline P1 X P4 & 16,622 & $\mathrm{P}=, 000$ \\
\hline P1 X P6 &, 041 & $\mathrm{P}=, 840$ \\
\hline P1 x P7 & 2,071 & $\mathrm{P}=, 150$ \\
\hline P1 X P8 & 1,721 & $\mathrm{P}=, 190$ \\
\hline P1 x P10 & 4,202 & $\mathrm{P}=, 040$ \\
\hline P1 X P12 & 7,607 & $\mathrm{P}=, 006$ \\
\hline P1 X P13 & 3,191 & $\mathrm{P}=, 203$ \\
\hline
\end{tabular}

\section{Discussão}

Em relação a correlação estatística para a dimensão cognitiva, observou-se uma concordância significativa entre os itens P3, P4 P10 e P12, corroborando com Leigh (2008), que afirma que a simulação potencializa o desempenho cognitivo dos estudantes, apontando a simulação como uma estratégia que permite que aprendizagem seja significativa. Um estudo Saudita com 117 estudantes de enfermagem também mostrou que a simulação como estratégia para a educação clínica promove a satisfação dos estudantes com a aprendizagem e melhora sua autoconfiança (Omer, 2016).

Ao analisarmos a dimensão cognitiva em relação ao desenvolvimento de competências para a tomada de decisões acertadas, item P13, neste estudo foi demonstrado que 98,9\% dos estudantes concordam completamente e concordam com essa afirmativa, no estudo na Escola Superior de Enfermagem de Coimbra, (ESEnfC) utilizando o mesmo instrumento para 
avaliação do debriefing os estudantes partilharam da mesma ideia, visto que $98,1 \%$ referiram ter desenvolvido competências para tomada de decisões acertadas (Coutinho et al., 2014).

Ainda em relação à dimensão cognitiva, um estudo realizado por Mazzo et al. (2019) na Universidade de São Paulo em 2016, com estudantes de medicina, a dimensão cognitiva obteve a maior média 4,5, aproximando-se da média que encontramos de 4,7 para essa dimensão em nosso estudo. Neste mesmo estudo os itens P3 “Aprender mais”, P12 "Identificar aspectos que devo melhorar em atuações futuras" e P13 "Desenvolver competências para tomada de decisões acertadas", também obtiveram destaque em relação ao nível de concordância, sendo de 85,8\% para P3, 80,8\% para P12 e 74,7\% para P13.

Relacionando a dimensão cognitiva na avaliação do debriefing, o estudo evidenciou total concordância com as Diretrizes Curriculares Nacionais dos Cursos de Graduação em Medicina (DCNs), que trata principalmente dos itens P3: Aprender Mais e P12: Identificar os aspectos que devem melhorar em atuações futuras. Assim, segundo Ministério da Educação [MEC] (2014), o Art 7º, inciso III da Resolução n ${ }^{\circ} 3$, de 20 de junho de 2014 traz: aprender interprofis sionalmente, com base na reflexão sobre a própria prática e pela troca de saberes com profissionais da área da saúde e outras áreas do conhecimento, para a orientação da identificação e discussão dos problemas, estimulando o aprimoramento da colaboração e da qualidade da atenção à saúde.

Acredita-se que a porcentagem de concordância deste estudo para os itens P3: 98,9\% e P12: 99,9\% estejam relacionadas com as características que metodologia ativa possui, instigando o pensamento crítico-reflexivo, e automonitoramento por meio de um envolvimento ativo. Ainda sob a ótica do Art $7^{\circ}$, inciso III da Resolução nº 3 , de 20 de junho de 2014: “Aprender em situações e ambientes protegidos e controlados, ou em simulações da realidade, identificando e avaliando o erro, como insumo da aprendizagem profissional e organizacional e como suporte pedagógico” (MEC, 2014).

Metodologia essa que permite explorar o ciclo de aprendizagem de Kolb (2014), onde a aprendizagem é compreendida como um processo de transformação da experiência, tendo como base etapas que fazem parte de um ciclo em que contempla a experiência, a reflexão, a conceituação abstrata e a experimentação, fazendo com que o estudante, durante a oportunidade do debriefing que representa a segunda e a terceira fase do ciclo, consiga atingir o senso crítico-reflexivo e consiga perceber-se enquanto agente do próprio saber, pois a partir das reflexões, do que foi possível realizar, dos acertos e dos erros, vê-se a possibilidade de buscar melhorar o conhecimento para as próximas oportunidades de atuação, onde se encontrará a quarta fase do ciclo com a experimentação, do que fora refletido, discutido e elaborado e embasado a partir da segunda e terceira fase, havendo assim a concretização do conhecimento.

Assim como o uso de simulação realística traz consigo a perspectiva behaviorista de avaliar as habilidades e competências dentro do terceiro nível da pirâmide de Miller (1990), a qual acontece por meio de simulações, não obstante é nessa ocasião que o estudante demonstra sua capacidade de tomada de decisão, realização de procedimentos, trabalho em equipe e raciocínio crítico-reflexivo, como visto no que tange o item P13.

Por conseguinte, ao analisar o item P13: Desenvolver competências para tomada de decisões acertadas o Art $7^{\circ}$, inciso III da Resolução nº 3, de 20 de junho de 2014 corrobora:

“Tomada de Decisões, com base na análise crítica e contextualizada das evidências científicas, da escuta ativa das pessoas, famílias, grupos e comunidades, das políticas públicas sociais e de saúde, de modo a racionalizar e aperfeiçoar a aplicação de conhecimentos, metodologias, procedimentos, instalações, equipamentos, insumos e medicamentos, de modo a produzir melhorias no acesso e na qualidade integral à saúde da população e no desenvolvimento científico, tecnológico e inovação que retroalimentam as decisões" (MEC, 2014).

De acordo com Boterf (2003), saber agir com pertinência se revela no profissional que tem iniciativa e toma decisões pertinentes, assumindo responsabilidades e inovando; é aquele que faz escolhas, assume riscos e reage a contingências, antecipando e prevendo as consequências; sabe interpretar, compreender e como agir diante de situações deterioradas, e por 
isso é capaz de resolver problemas. Esta competência possibilita a mobilização dos saberes com o fato de agir com pertinência para aprender a aprender e envolver-se com o contexto.

\section{Conclusão}

O debriefing mostrou ser uma metodologia de avaliação de acordo com as DCNs recomendadas para a formação dos cursos de graduação em medicina, capaz de avaliar o conhecimento cognitivo dos estudantes, além de estimular e contribuir para o desenvolvimento de competências e habilidades necessárias para o exercício profissional da medicina. Os níveis de concordância na dimensão foram altos, demonstrando a efetividade do instrumento de avaliação do debriefing e que a percepção do aluno é clara em relação a potencialidade da metodologia, sendo o nível de satisfação em relação ao debriefing bastante elevado.

Uma das maiores limitações do estudo refere-se a escassez da aplicabilidade do instrumento de avaliação do debriefing, em especial na simulação realística em estudantes de graduação em medicina, visto que o instrumento foi recentemente validado no país e somente foi aplicado nos cursos de graduação em enfermagem. Assim, sugere-se que novos estudos utilizando o instrumento da EADaS sejam realizados, em outros cursos de graduação em medicina com simulação realística de alta fidelidade e, assim; que o ineditismo para o estudo sirva de contribuição para fortalecimento de uma metodologia ativa considerada efetiva para o processo de ensino e aprendizagem.

\section{Referências}

Almeida, R. G. d. S., Mazzo, A., Martins, J. C. A., Coutinho, V. R. D., Jorge, B. M., \& Mendes, I. A. C. (2016). Validação para a língua portuguesa da Debriefi ng Experience Scale. Revista Brasileira de Enfermagem, 69(4), 705-711. https://doi.org/10.1590/0034-7167.2016690413i

Boterf, G. L. (2003). Desenvolvendo a competência dos profissionais (3 $\left.{ }^{\mathrm{a}} \mathrm{ed}.\right)$. Penso.

Brandão, C. S., Collares, C. F., \& Marin, H. F. (2014). A simulação realística como ferramenta educacional para estudantes de medicina. Scientia Medica, 24(2), 187. https://doi.org/10.15448/1980-6108.2014.2.16189

Costa, R. R. d. O., Medeiros, S. M. d., Coutinho, V. R. D., Veríssimo, C. M. F., Silva, M. M. M. d., \& Lucena, E. E. d. S. (2020). Simulação clínica no desempenho cognitivo, satisfação e autoconfiança na aprendizagem: Estudo quase-experimental. Acta Paulista de Enfermagem, 33. https://doi.org/10.37689/acta-ape/2020ao01236

Coutinho, V. R. D., Martins, J. C. A., \& Pereira, M. d. F. C. R. (2014). Construção e validação da escala de avaliação do debriefing associado à simulação (EADaS). Revista de Enfermagem Referência, 4(2), 41-50. https://doi.org/10.12707/riii1392

Esperón, J. M. T. (2017). Quantitative research in nursing science. Escola Anna Nery - Revista de Enfermagem, 21(1). https://doi.org/10.5935/14148145.20170027

Ferreira, R. P. N., Guedes, H. M., Oliveira, D. W. D., \& Miranda, J. L. d. (2018). Simulação realística como método de ensino no aprendizado de estudantes da área da saúde. Revista De Enfermagem Do Centro-Oeste Mineiro, 8. https://doi.org/10.19175/recom.v8i0.2508

Kolb, D. A. (2014). Experiential learning: Experience as the source of learning and development (2 $2^{\mathrm{a}}$ ed.). Prentice-Hall.

Leigh, G. T. (2008). High-Fidelity patient simulation and nursing students' self-efficacy: A review of the literature. International Journal of Nursing Education Scholarship, 5(1), 1-17. https://doi.org/10.2202/1548-923x.1613

Lovato, F. L., Michelotti, A., \& Da Silva Loreto, E. L. (2018). Metodologias ativas de aprendizagem: Uma breve revisão. Acta Scientiae, 20(2). https://doi.org/10.17648/acta.scientiae.v20iss2id3690

Marcomini, E. K., Martins, E. S., Lopes, N. V., Paula, N. V. K. d., \& Liberati, B. A. d. S. (2017). Influência da simulação realística no ensino e aprendizado da enfermagem. Varia Scientia, 3(2), 1-8. https://doi.org/10.48075/vscs.v3i2.17687

Mazzo, A., Franzon, J. C., Meska, M. H. G., Machado, G. C. C., Coutinho, V. R. D., \& Pereira Junior, G. A. (2019). Implications on the use of sound and image in debriefing assessment. Reme Revista Mineira de Enfermagem, 23. https://doi.org/10.5935/1415-2762.20190007

Miller, G. E. (1990). The assessment of clinical skills/competence/performance. Academic Medicine, 65(9), S63-7. https://doi.org/10.1097/00001888199009000-00045

Omer, T. (2016). Nursing students' perceptions of satisfaction and self-confidence with clinical simulation experience. Journal of Education and Practice, 7(5), 131-138.

Pedroso, J. d. S., Silva, K. S. d., \& Santos, L. P. d. (2017). Pesquisa descritiva e pesquisa prescritiva. Jicex, 9(9). 
Resolução n. 3, de 20 de junho de 2014 (2014). Institui Diretrizes Curriculares Nacionais do Curso de Graduação em Medicina e dá outras providências. Diário Oficial da União. Brasília, DF: Ministério da Educação.

Rodrigues, F. L., Moura, L. M. d., Boeckmann, L. M. M., Melo, M. C., França, F. C. d. V., \& SantAna, G. S. (2020). Avaliação do processo ensino e aprendizagem no ambiente de simulação realística na graduação em enfermagem. Enfermagem em Foco, 10(6). https://doi.org/10.21675/2357707x.2019.v10.n6.2782

Vaccari, A., Farias, G. F., \& Porto, D. S. (2020). Implementation of a lesson plan model in the nursing laboratory: Strengthening learning. Revista Gaúcha de Enfermagem, 41. https://doi.org/10.1590/1983-1447.2020.20190174

Valadares, A. F. M., \& Magro, M. C. d. S. (2014). Opinião dos estudantes de enfermagem sobre a simulação realística e o estágio curricular em cenário hospitalar. Acta Paulista de Enfermagem, 27(2), 138-143. https://doi.org/10.1590/19820194201400025

Waterkemper, R., \& Prado, M. L. d. (2011). Estratégias de ensino-aprendizagem em cursos de graduação em Enfermagem. Avances en Enfermería, 29(2), $234-246$.

Ypinazar, V., \& Margolis, S. (2006). Clinical simulators: Applications and implications for rural medical education. Rural and Remote Health. https://doi.org/10.22605/rrh527 